\title{
Editorial: The Role of RNA in Genome Stability: To Wreck or Repair?
}

\author{
Belén Gómez-González ${ }^{1,2 *}$, Arijit Dutta ${ }^{3 *}$ and Wenyi Feng ${ }^{4 *}$ \\ ${ }^{1}$ Departamento de Genética, Facultad de Biología, Universidad de Sevilla, Seville, Spain, ${ }^{2}$ Centro Andaluz de Biología Moleculary \\ Medicina Regenerativa-CABIMER, Universidad de Sevilla-CSIC, Seville, Spain, ${ }^{3}$ Department of Biochemistry and Structural \\ Biology, University of Texas Health Science Center at San Antonio, San Antonio, TX, United States, ${ }^{4}$ Department of Biochemistry \\ and Molecular Biology, SUNY Upstate Medical University, Syracuse, NY, United States
}

Keywords: DNA damage repair, genome stability, R-loop, DNA-RNA hybrid, transcription, DSB repair

Editorial on the Research Topic

The Role of RNA in Genome Stability: To Wreck or Repair?

It has long been known that the process of transcription is a source of genetic instability. RNA molecules have been implicated in both the generation of DNA damage and, in the recent years, in its repair. These seemingly conflicting roles of RNA motivated us to open this research topic covering transcription-associated genetic instability sources and the role of RNA in the repair of DNA damage.

The genome is challenged by both endogenous and exogenous sources of DNA damage. Additionally, failures in DNA metabolic processes, such as replication impairments or chromosomal miss-segregation, can threaten the transmission of the genetic information to the offspring by causing genome and chromosome instability. In particular, DNA transcription increases the frequency of mutation and recombination; this latter being caused, although not exclusively, by increased number of DNA double-strand breaks (DSBs), which are among the most harmful DNA lesions (Gaillard and Aguilera, 2016). RNA transcripts are constantly generated to supply for protein synthesis, RNA interference pathways, and regulation of transcription, translation, splicing as well as DNA repair processes via non-coding RNAs. Even heterochromatic regions can generate RNA molecules at telomeres (Azzalin et al., 2007; Luke et al., 2008; Schoeftner and Blasco, 2008) and centromeres (Saffery et al., 2003), that participate in the regulation of the telomeric (Fukagawa et al., 2004) and centromeric structures, thus impacting chromosome segregation and chromosome instability. The diverse roles of human centromeric RNA in chromosome stability were compiled by Leclerc and Kitagawa in this Frontiers Topic.

Nascent RNA molecules can potentially re-anneal with the DNA template to form DNA-RNA hybrids within ORFs (Huertas and Aguilera, 2003) and at transcription termination regions (Mischo et al., 2011) but also in telomeric (Luke et al., 2008) or centromeric regions (Kabeche et al., 2018; Mishra et al., 2021) and it is well established that DNA-RNA hybrids and R-loops, formed by the hybrid and the displaced single stranded DNA, are a major source of replication fork problems and ultimately DSBs (Gomez-Gonzalez and Aguilera, 2019). Thus, the odds of a transcribing DNA molecule to suffer DSBs are higher than a non-transcribing equivalent. In this Frontiers Topic, Long et al. describe how DSBs within a transcribed DNA causes a transient transcriptional shut-down but, at the same time, the DSB-induced transcripts are produced de novo and participate in DNA damage signaling (Francia et al., 2012; Michelini et al., 2017). The context in which the DNA breaks occur can affect the outcome of the repair mechanisms. Most of this effect is likely due to transcriptionmediated changes in the chromatin status (Aymard et al., 2014; Clouaire and Legube, 2015). Notwithstanding, the potential impact of RNA molecules on DSB repair is emerging as a new controversial, but fascinating, field of study. 
For instance, RNA molecules can impact DNA damage repair directly or indirectly by recruiting new protein factors involved in DNA damage signaling or repair. In fact, an increasing number of RNA-binding proteins appear to have a role in the DNA damage response, specifically to DSBs, as examined here by Klaric et al. Most of them were already studied for their functions in RNA metabolism, with the expected involvement in gene expression, but recently have been re-discovered to play additional functions in the regulation of DSB signaling and repair. It is of note that several RNA-binding proteins have an intrinsically disordered region (IDR), such as that in the Fragile $\mathrm{X}$ mental retardation protein (FMRP), which can function as an R-loop reader as shown by Dettori et al. in this Frontiers topic (Dettori et al.). Moreover, IDRs could facilitate dynamic assembly of protein complexes including R-loop resolvases such as DHX9 via liquidliquid phase separation to promote R-loop resolution.

Adding a new layer of complexity, the affinity of RNA-binding proteins for RNA can be modulated by different posttranscriptional chemical modifications of the RNA molecule, creating a plethora of possible combinatorial epi-transcriptomic signatures that offer a high-level regulation to the DNA damage response. A prime example would be the N6-Methyladenosine (m6A), which, as reviewed by $\mathrm{Qu}$ et al. and Jimeno et al., modulates repair and genome stability ( $\mathrm{Qu}$ et al.; Jimeno et al.). These review articles have summarized the recent findings on the role of RNA modifiers, such as RNA methyltransferase METTL3 and m6A RNA reader YTHDC1, in promoting DSB repair via homologous recombination (Zhang et al., 2020). Furthermore, the RNA molecule can also be edited, and A-to-I deamination via ADAR2 can also directly influence DSB repair by promoting R-loop resolution, facilitating DNA end resection to initiate homology-dependent repair (Jimeno et al., 2021a).

A growing number of reports have revealed that DSBs stimulate the formation of DNA-RNA hybrids (Aguilera and Gomez-Gonzalez, 2017) and some RNA-binding proteins can counteract or unwind the formation of hybrids at DSBs, as shown for Senataxin or DDX5 helicases among others (Cohen et al.,

\section{REFERENCES}

Aguilera, A., and Gómez-González, B. (2017). DNA-RNA Hybrids: the Risks of DNA Breakage during Transcription. Nat. Struct. Mol. Biol. 24, 439-443. doi:10.1038/nsmb.3395

Aymard, F., Bugler, B., Schmidt, C. K., Guillou, E., Caron, P., Briois, S., et al. (2014). Transcriptionally Active Chromatin Recruits Homologous Recombination at DNA Double-Strand Breaks. Nat. Struct. Mol. Biol. 21, 366-374. doi:10.1038/ nsmb.2796

Azzalin, C. M., Reichenbach, P., Khoriauli, L., Giulotto, E., and Lingner, J. (2007). Telomeric Repeat-Containing RNA and RNA S] urveillance Factors at Mammalian Chromosome Ends. Science 318, 798-801. doi:10.1126/science.1147182

Clouaire, T., and Legube, G. (2015). DNA Double Strand Break Repair Pathway Choice: a Chromatin Based Decision. Nucleus 6, 107-113. doi:10.1080/ 19491034.2015.1010946

Cohen, S., Puget, N., Lin, Y.-L., Clouaire, T., Aguirrebengoa, M., Rocher, V., et al. (2018). Senataxin Resolves RNA:DNA Hybrids Forming at DNA DoubleStrand Breaks to Prevent Translocations. Nat. Commun. 9, 533. doi:10.1038/ s41467-018-02894-w

D’Alessandro, G., Whelan, D. R., Howard, S. M., Vitelli, V., Renaudin, X., Adamowicz, M., et al. (2018). BRCA2 Controls DNA:RNA Hybrid Level at
2018; Yu et al., 2020) (see Klaric et al. for a complete review). Strikingly, the DNA damage response can take advantage of this ability to promote efficient DNA damage repair, as exemplified by BRCA2 promoting DDX5 activity at damaged DNA (Sessa et al., 2021) or by BRCA1 interacting with Senataxin and the RNAi machinery to promote the repair of nicks at transcription termination pause sites (Hatchi et al., 2021). However, the role of DNA-RNA hybrids at DSBs remains controversial (Marnef and Legube, 2021). Whereas it has been put forth that accidental formation of the DNA-RNA hybrids at DSBs can interfere with homologous recombination repair (Ortega et al., 2021), it has also been suggested that hybrids are intermediates required for repair (Keskin et al., 2014; Ohle et al., 2016; D’Alessandro et al., 2018; Lu et al., 2018; Liu et al., 2021; Ouyang et al., 2021).

This Frontiers topic covers the most recent findings concerning the roles of RNA in genome stability and DNA repair mechanisms. We hope that the readers will find this collection of articles educational and useful for their own study to further advance this emerging field of research.

\section{AUTHOR CONTRIBUTIONS}

BG-G wrote this editorial introduction with AD's contribution, and revision by WF. All authors agreed on the submitted version. $\mathrm{AD}$ initially conceived this research topic and set up the editorial team with BG-G and WF. All the editors collaboratively edited the manuscripts, that were subjected to peer-review prior to acceptance.

\section{ACKNOWLEDGMENTS}

We thank all authors of the papers published in this research topic for their valuable contributions and the referees for their rigorous and on time review. We also thank Elsa Zacco for acting as editor for one paper in the research topic.

DSBs by Mediating RNase H2 Recruitment. Nat. Commun. 9, 5376. doi:10. 1038/s41467-018-07799-2

Francia, S., Michelini, F., Saxena, A., Tang, D., de Hoon, M., Anelli, V., et al. (2012). Site-specific DICER and DROSHA RNA Products Control the DNA-Damage Response. Nature 488, 231-235. doi:10.1038/nature11179

Fukagawa, T., Nogami, M., Yoshikawa, M., Ikeno, M., Okazaki, T., Takami, Y., et al. (2004). Dicer Is Essential for Formation of the Heterochromatin Structure in Vertebrate Cells. Nat. Cel Biol 6, 784-791. doi:10.1038/ncb1155

Gaillard, H., and Aguilera, A. (2016). Transcription as a Threat to Genome Integrity. Annu. Rev. Biochem. 85, 291-317. doi:10.1146/annurev-biochem-060815-014908

Gómez-González, B., and Aguilera, A. (2019). Transcription-mediated Replication Hindrance: a Major Driver of Genome Instability. Genes Dev. 33, 1008-1026. doi:10.1101/gad.324517.119

Hatchi, E., Goehring, L., Landini, S., Skourti-Stathaki, K., DeConti, D. K., Abderazzaq, F. O., et al. (2021). BRCA1 and RNAi Factors Promote Repair Mediated by Small RNAs and PALB2-RAD52. Nature 591, 665-670. doi:10. 1038/s41586-020-03150-2

Huertas, P., and Aguilera, A. (2003). Cotranscriptionally Formed DNA:RNA Hybrids Mediate Transcription Elongation Impairment and Transcription-Associated Recombination. Mol. Cel 12, 711-721. doi:10.1016/j.molcel.2003.08.010

Jimeno, S., Prados-Carvajal, R., Fernández-Ávila, M. J., Silva, S., Silvestris, D. A., Endara-Coll, M., et al. (2021a). ADAR-mediated RNA Editing of DNA:RNA 
Hybrids Is Required for DNA Double Strand Break Repair. Nat. Commun. 12, 5512. doi:10.1038/s41467-021-25790-2

Kabeche, L., Nguyen, H. D., Buisson, R., and Zou, L. (2018). A Mitosis-specific and R Loop-Driven ATR Pathway Promotes Faithful Chromosome Segregation. Science 359, 108-114. doi:10.1126/science.an6490

Keskin, H., Shen, Y., Huang, F., Patel, M., Yang, T., Ashley, K., et al. (2014). Transcript-RNA-templated DNA Recombination and Repair. Nature 515, 436-439. doi:10.1038/nature13682

Liu, S., Hua, Y., Wang, J., Li, L., Yuan, J., Zhang, B., et al. (2021). RNA Polymerase III Is Required for the Repair of DNA Double-Strand Breaks by Homologous Recombination. Cell 184, 1314-1329. doi:10.1016/j.cell.2021.01.048

Lu, W.-T., Hawley, B. R., Skalka, G. L., Baldock, R. A., Smith, E. M., Bader, A. S., et al. (2018). Drosha Drives the Formation of DNA:RNA Hybrids Around DNA Break Sites to Facilitate DNA Repair. Nat. Commun. 9, 532. doi:10.1038/ s41467-018-02893-x

Luke, B., Panza, A., Redon, S., Iglesias, N., Li, Z., and Lingner, J. (2008). The Ratlp $5^{\prime}$ to $3^{\prime}$ Exonuclease Degrades Telomeric Repeat-Containing RNA and Promotes Telomere Elongation in Saccharomyces cerevisiae. Mol. Cel 32, 465-477. doi:10.1016/j.molcel.2008.10.019

Marnef, A., and Legube, G. (2021). R-loops as Janus-Faced Modulators of DNA Repair. Nat. Cel Biol 23, 305-313. doi:10.1038/s41556-021-00663-4

Michelini, F., Pitchiaya, S., Vitelli, V., Sharma, S., Gioia, U., Pessina, F., et al. (2017). Damage-induced lncRNAs Control the DNA Damage Response through Interaction with DDRNAs at Individual Double-Strand Breaks. Nat. Cel Biol 19, 1400-1411. doi:10.1038/ncb3643

Mischo, H. E., Gómez-González, B., Grzechnik, P., Rondón, A. G., Wei, W., Steinmetz, L., et al. (2011). Yeast Sen1 Helicase Protects the Genome from Transcription-Associated Instability. Mol. Cel 41, 21-32. doi:10.1016/j.molcel. 2010.12.007

Mishra, P. K., Chakraborty, A., Yeh, E., Feng, W., Bloom, K. S., and Basrai, M. A. (2021). R-loops at Centromeric Chromatin Contribute to Defects in Kinetochore Integrity and Chromosomal Instability in Budding Yeast. MBoC 32, 74-89. doi:10.1091/mbc.e20-06-0379

Ohle, C., Tesorero, R., Schermann, G., Dobrev, N., Sinning, I., and Fischer, T. (2016). Transient RNA-DNA Hybrids Are Required for Efficient DoubleStrand Break Repair. Cell 167, 1001-1013. doi:10.1016/j.cell.2016.10.001

Ortega, P., Mérida-Cerro, J. A., Rondón, A. G., Gómez-González, B., and Aguilera, A. (2021). DNA-RNA Hybrids at DSBs Interfere with Repair by Homologous Recombination. Elife 10. doi:10.7554/eLife.69881
Ouyang, J., Yadav, T., Zhang, J.-M., Yang, H., Rheinbay, E., Guo, H., et al. (2021). RNA Transcripts Stimulate Homologous Recombination by Forming DRLoops. Nature 594, 283-288. doi:10.1038/s41586-021-03538-8

Saffery, R., Sumer, H., Hassan, S., Wong, L. H., Craig, J. M., Todokoro, K., et al. (2003). Transcription within a Functional Human Centromere. Mol. Cel 12, 509-516. doi:10.1016/s1097-2765(03)00279-x

Schoeftner, S., and Blasco, M. A. (2008). Developmentally Regulated Transcription of Mammalian Telomeres by DNA-dependent RNA Polymerase II. Nat. Cel Biol 10, 228-236. doi:10.1038/ncb1685

Sessa, G., Gómez-González, B., Silva, S., Pérez-Calero, C., Beaurepere, R., Barroso, S., et al. (2021). BRCA2 Promotes DNA-RNA Hybrid Resolution by DDX5 Helicase at DNA Breaks to Facilitate Their Repairł. EMBO J. 40, e106018. doi:10.15252/embj.2020106018

Yu, Z., Mersaoui, S. Y., Guitton-Sert, L., Coulombe, Y., Song, J., Masson, J. Y., et al. (2020). DDX5 Resolves R-Loops at DNA Double-Strand Breaks to Promote DNA Repair and Avoid Chromosomal Deletions. NAR Cancer 2, zcaa028. doi:10.1093/narcan/zcaa028

Zhang, C., Chen, L., Peng, D., Jiang, A., He, Y., Zeng, Y., et al. (2020). METTL3 and N6-Methyladenosine Promote Homologous Recombination-Mediated Repair of DSBs by Modulating DNA-RNA Hybrid Accumulation. Mol. Cel 79, 425-442. doi:10.1016/j.molcel.2020.06.017

Conflict of Interest: The authors declare that the research was conducted in the absence of any commercial or financial relationships that could be construed as a potential conflict of interest.

Publisher's Note: All claims expressed in this article are solely those of the authors and do not necessarily represent those of their affiliated organizations, or those of the publisher, the editors and the reviewers. Any product that may be evaluated in this article, or claim that may be made by its manufacturer, is not guaranteed or endorsed by the publisher.

Copyright () 2022 Gómez-González, Dutta and Feng. This is an open-access article distributed under the terms of the Creative Commons Attribution License (CC BY). The use, distribution or reproduction in other forums is permitted, provided the original author(s) and the copyright owner(s) are credited and that the original publication in this journal is cited, in accordance with accepted academic practice. No use, distribution or reproduction is permitted which does not comply with these terms. 